\title{
OBSERVATIONS SUR LA PISCICULTURE ARTIFICIELLE DU BROCHET
}

\author{
par JEAN PORTAL \\ Ingénieur des Travaux publics de l'Etat \\ a Rennes (Hle-et-Vilaine)
}

Au cours des années r 944 à 1947 le service des Ponts ct Chaussées a elfectué à la l'isciculture du Boulet, située sur le territoire de la commune de Feins (Ille-et-Vilaine), quelques essais et expériences sur le Brochet.

La Pisciculture du Boulet a été aménagéc depuis 1942, sur des dépendances du caual d'Ille et Rance, en vue de la production d'alevins de poissons blancs, destinés au réempoissonnement dẹ cours d'eau du Momaine Public du département d'Ille-et-Vilaine.

Les demandes d'alevins de Brochets des Sociétés de pêche sont nombreuses. Jusqu'à présent il a été impossible d'y donner satisfaction, ces Jomandes dépassant très largocment la production annuelle de la piscirulture. Les quantités de Brochctons obtenues, chaque amméc, dans l'étangr de Planche-Roger utilisé à cet effet, sont très variables, en tour ras toujours incertaines, sans qu'il soit possible encore d'en déterminer les raisons. Ceci nous a conduit à tenter d'accoilse rette production, en effectuant la lécondation el l'incubation artifirielle.

Il convenait, tout d'aborl, d'essayer de conserver en caplivité des reproducteurs, afin d'ctre en mestre d'effectuer les opérations de fécondation arlificielle aiscoment, le moment venu. Jusqu à présent nous n'avons pu sésonalre ce problème, et toutes les expériences tentées pour y parrenir ont échoué.

Ia conservation des Brochels, dans les bassins de la Pisciculture, s est révélée impossible. Ces hassins présentent les dimensions suivantes : - mètres $\times 2 \mathrm{~m}$. 70,4 mèl res $\times 3 \mathrm{~m}$. 3o, avec une profoncleur d'eau de " $\mathrm{m}$. $7^{5}$. Mème en couvrant ces bassins de filets de camouflage, imime eu y jetant des alevins de Gardons ot de Carpes, il $n$ 'a jamais été possible d'y conserver les Brochels plus d'une quinzane de jours. Au-delà de re dẹlai, ils dépérissent et meurent. Ils se blessent généralement entrc eux un rontre les parois et no consomment pas la nourriture qui est mike à leur disposition.

Il en est de même des manches de couteaux et poignards, qui se dévorent entre cux, le mangeur crevant comme le mangé. Il esl à peu pres impossible de les conserver plus de 3 ou 4 semaines dans les bassins, sous peine de courir le risque d'avoir des pertes considérables. 
Un petit bassin du' système "Dubisch " de 4 mètres $\times 6$ mètres, d'une profondeur moyenne de o m. 3o, avec fond gazonné, a été aménagé en 1944. En automne 1945, après la pèche de l'étang de Planche-Roger, nous y avons placé 7 Brochets reproducteurs de 3 à 4 livres. Ils s y sont conservés dans des conditions convenables pendant un mois, suns toutefois accepter la nourriture d'alevins de Gardons mise à lear disposition. Le bassin étant garni d'herbes, ils s'y cachaient et restaient immobiles des journées entières.

Toutefois, nous avions placé autour du bassin des toiles à sac, formant un paravent de I mètre de hauteur, ce qui masquait les Brochets à a vi vin des passants. Cette circonstance nous a paru favorable en procurant aux Brochets une sorte de tranquilité. Malgré cela il est certain qu'une capti. vité plus longue, aurait conduit à leur perte. L'un d'eux, le plus gros, portait au moment de la reprise une plaie profonde sur le flanc, près de la nageoire caudale. Mis en étang, cette blessure s'est très bien cicatrisée, ainsi que nous avons pu le constater à la pèche en automne 1946 .

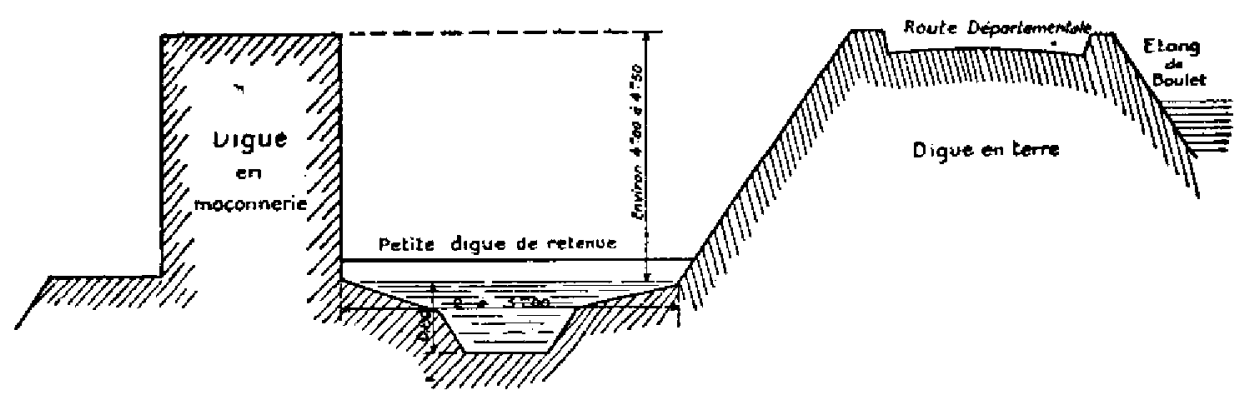

Fig. 12. - Coupe en travers de la frayère à Brochets entre les deux digues de l'étang du Boulet.

Entre les deux digues de l'étang du Boulet, nous avions installé en r944́ une sorte de frayère, présentant une longueur d'une quarantaine de mètres, une largeụr de 2 à 3 mètres et une profondeur de o $\mathrm{m}$. 50 à $\mathrm{om}$. 8o. I.e croquis ci-dessous explirque la siluation des lieux, mieux qu'une longue description. Lalimentation en eau était abondante. En deux endroits nous avions disposé au-dessus de l'eau des branchages formant cachette, où les Brochets se tenaient d'ailleurs le plus souvent. Le ro Mars I 95 , nous y avons placé deux femelles et trois mâles. La température de l'eau était de 8 à ro decrrés, donc favorable. Le ig Mars nous avons trouvé une femelle crevée, quelquès jours après un mâle. Le mâle, ni la femelle n'étaient à maturité. Nous avons dú retirer ceux qui restaicnt sous peine de les voir périr à leur tour. 
Vers la mi-Février 1944, dans les deux ruisseaux alimentant l'étang de Planche-Roger, nous avions isolé, au moyen de grilles, des longueurs de roo à r 50 mètres dans lesquelles nous avons placé des reproducteurs. Dès la fin du mois, nous avons pû observer que ces reproducteurs se lenaient cachés dans les herbes du bord ou du fond et demeuraient continuellement à la même place. Au bout de trois semaines, ils n'avaient pas frayé, paraissaient languissants et n'étaient pas à maturité. Ils se laissaien، capturer avec une épuisette, presque sans difficulté. Finalement, pour pe pas risquer de les perdre, nous avons dû enlever les grilles aval. Deux jours après ils avaient disparu et étaient passés dans l'étang.

Sur la rive nord de l'étang de Planche-Roger, existe une rigole nalurelle de $\mathbf{2} \mathbf{m}$. 5o de largeur, o m. 5o à $0 \mathrm{~m}$. 80 de profondeur. Cette rigole est à exposition sud, abritée dẹ venls du Nord par la lorèt voisine. Nous l'avions aménagée en I944, en rigole frayère, sur i I 7 mètres de longueur, au moyen de pẹtites vannes disposées à chatue extrémité. Ces vannes, volontairement, n'étaient pas étanches. L'eau de la rigole communiquait ainsi avec celle de l'étangr. Nous y avons immergé, le $2 \overline{0}$ Mars, à 16 heures, 28 Brochets reproducteurs. Le 7 Avril, le garde y trouve un Brochet mort. Lexamen de ce Brochet ne permet pas de découvrir la cause de cette perte. Il s'agissait d'une femelle ayant encore ses ceufs non parvenus à mafurité. Le i I Avril nous trouvons encore trois Brochels morts et de crainte de les voir tous disparaitre de la sorte, nous levons les vannes de communication avec l'étang. Le is Avril, un cinquième Bnochet apparait à la surface. Sur ces cinq Brochets, il $y$ avait trois femelles' pleines d'oufs et deux mâles n'ayant plus de laitance. Aucune trace de blessure n'a élé relevée et l'analyse de l'échantillon d'eau, emportée le i I Avril, n'a révélé absolument rien d'anormal. On est donc conduit à admettre que la mortalité constatée provient d'une cause d'ordre physiologique. Après le 12 Avril, les Brochets ont disparu de la rigole frayère et ont passés dans l'étang. Il n'y a plu de mortalité.

De l'étang du Boulet part une rigole servant à l'alimentation du canal d'Ille et Rance. Cette rigrole présente une largeur de 3 mètres en tête, r mètre au plafond avec I mètre de profondeur. Nous avons aménagé, dans cette rigole, des bassins de 25 mètres de longueur, séparés entre eux par de simples grillages. La encore, la conservation des Brochets n'a jamais donné des résultats intéressants. Ils se blessent entre eux, paraissent se nourrir très mal, mème s'ils sont en petit nombre (6 au maximum) dans chaque bassin. Jusqu'à présent, dans loutes les tentatives que nous avons faites, lorsque la durée de conservation dans ces bassins dépasse un mois et demi, la perte atteint $7^{\circ} \%$ pour les mâles. Elle est moindre pour les femelles, mais ces dernières ne parviennent jamais à maturité. 
Immédiatement en aval des bassins de la Pisciculture, un petit étang de 2 ares a été obtenu par construction d'une digue en travers du ruisseau du Boulet. Nous avons également essayé d'y conserver des reproducteurs femelles. Six femelles y ayant été placées le 4 Janvier 1947, l'une d'elle ayant crevé entre temps, nous avons retiré, le 13 Mars, les cinq femelles restantes. Elles étaient exactement dans le même état que celui du 4 Janvier, c'cst-à-dire qu'elles étaient très loin d'être parvenues à maturité, alors que les Brochets de l'étang du Boulet, vivant à l'état sauvage, étaient en période de fraie à ce moment lá.

Devant l'impossibilité d'obtenir en captivité des reproducteurs mâles et femelles à maturité, nous avons essayé d'en capturer à l'état sauvage. L'étang de Planche-Roger communique avec celui du Boulet par un pont donnant passage à une route départementale. La bouchure est constituée par un barrage à poutrelles surmonté d'une grille. A un mètre en amont de ce barrage, est disposée une vanne, avec de chaque côté des planchettes mobiles. Au cours des neuf premiers jours du mois de Mars I9/7, la température, qui avait été jusqu à ce moment lia très froide, s'est adoucie prögressivement et la pluie a commencé à tomber faisant fondre la glace qui recouvrait les étangs. Le débit des affuents, qui était faible, a augmenté progressivement. Cet adoucissement de température faisait espérer que la fraie du Brochet allait commencer sous peu. Le niveau dans l'étang du Boulet élait le mème que dans l'étang de Planche-Roger. La grille sur le barrage à poutrelles émergeait de o m. 50 . Le garde, dès le to Mars, a exercé une surveillance à cette grille afin de s'assurer que les Brochets de l'étang du Boulet ne cherchaient pas à remonter dans celui de PlancheRoger. Or, dès le ro, des passants signalaient que des Brochets avaient été vus le nez à la grille. Les $I$, 12 et i3 Mars, le grarde capturait avé une simple épuisette, immédiatement en aval de la grille, sous le pont, ro femelles et trois mâles à maturité. Ces poissons ont été emportẹ́s vivants a la Pisciculture où ils on tété utilisés pour des essais de fécondation artificielle.

Ces opérations ont été faites dans les conditions suivantes :

Le jeudi i3 Mars sont utilisés trois femelles et sept mâles. La manipulation est opérćc par le garde pisciculteur et un garte féféral. Les fomelles qui sont à maturité donnent bien leurs oufs qui sont recueillis soigneusement à sec dans trois bols. Les mâles donncnt ensuite peu de laitance, une à deux grouttes seulement pour chacun. Nous remarquons que les mâles captifs donnent mieux leur laitance quèe ceux pris à l'état sauvage. Les mâles semblent se contracter et retenir leur laitance. Les gouttes de laitance tombent directẹment sur les oufs. Le mélange d'oufs et laitance est fait aussitôt avec une plume, puis au bout de 5 à 6 minutes, le tout est 
versé dans un bol d'eau, pctit à pêtit en agitant au fur et à mesure. Après quelques minutes d'attente les cufs sont lavés et mis dans une carafe de Lug, avec un courant d'cau provoquant le brassage des oufs. Au bout d'un quart d'heure, nous observons qu'un assez grand nombre d'oufs deviennent blancs et opaques. Que s'est-il passé ?

Au moment de la fécondation, qui a été faite à l abri, dans un petit local et derrière une baie vitrée, un rayon chaud de soleil est venu réchauffer l'eau dans les bols dont la température s'est trouvée devenir supérieure d'au mois 2 à 3 degré à celle de la carafe de Zug, placée dehors, qui était à 7 degré. Celte variation de température a certainement élé néfaste à un grand nombre d'wufs. Nous avons, dans dautres conditions que nous indiquons plus loin, effectué une observation analogue. Ces cufs, placés dans deux carafes de Zug n'ont pas donné de résultats. A notre avis le trarde a laissé un courant d'eau trop fort, ce qui a provoqué un brassage Irop énergique et une destruction des ocufs. La quantité de laitance était vraisemblablement insuffisante.

Le samedi í Mars, une deuxième opération a été faite par nous-même en utilisant uṇe seule femelle et trois mâles. L un des mâles ạvait été capturé dans la pecherie d'anguilles. Il était presque mort parce qu'ayant passé dans les vannes, eñtraîné par le courant. Ce mâle nous a donné cinq gouttes de laitance. Deux autres, déjà employés le i 3 Mars donnent chacun une ou deux gouttes. La fécondation est faite à sec. Nous prenons soin de bien laver les œufs dans de l'eau à la même température que celle de la carafe de Zug. Au moment ou nous déversons les weufs dans la carafe, nous remarquons qu'ils se collent aux parois ou sc rassemblent en paquets. Nous mettons un courant d'eau très faible. Pour éviter aux ocuf́s de se coller à la paroi nous les remuons légèrement avec une plume. Petit à petit le mucus disparait, les coufs deviennent libres au bout de deux heurcs. Il y a très peu d'ceufs non fécondés, quelques centaines sur environ 5 à 6.ooo. L'opération parait avoì parfaitement réussi. Pendant l'incubation il y a également très peu de mortalité. Le 27 Mars lẹs ocufs sont transférés dans un bac d'incubation d'oufs de Truites, sur des clizies, qui ne sont autres que des petites caissettes utilisées pour le transport des civelles, dont le fond est fait de toile fine (soic à bluter). Les 28 et 29 Mars se produit une éclosion générale Le 2 Avril la mortalité demeure insignifiante. La résorption de la vésicule s'effectuant normalement, nous emportons les alevins daṇs l'étang du Malavisé, de 2 hectares, où il n'y a aucun Brochet.

Dans les caisseties à civelles, placées dans l'eau du bac d'incubation, les alevins de Brochets, après l'éclosion, restent couchés sur le fond ou accrochés presque verticalement aux parois de la caissette. 
Samedi i5 Mars nous avons emporté le Brochet mâle mort. Nous lui avons enlevé les testicules et ainsi nous avons pu remarquer qüils restaient gorgés de laitance. Pressurés ces teslicules nous en donnent une cuillerée à café, ce qui est une quanlité susceptibje de léconder les. œuf́s de plusieurs fenelles. Il semble donc inexact de dire que plusieurs mâles sont nécessaires pour une seule femelle. A notre avis, un màle possède suffisamment de laitance pour féconder un nombre d'ơufs considérable. La difficulté est de lui faire donner cette laitance.

Le dimanche i6 Mars nous rapportons à Rennes, de la Pisciculture, une femelle à maturité et un mâle. Ces sujets ont été pris à 15 heures et emportés à sec sans aucune précaution spéciale. Lundi I 7 Mars, entre ${ }^{3} 3$ heures et $\mathbf{3}$ h. 3o, nous effectuons la fécondation artificielle, à sec, des cuis de celte femelle et procédons de la façon suivante.

Les oufs de la femelle sont obtenus facilement par simple pression sur le ventre. Ils sont recuejllis dans un bol. Ensuite, nous relirons les testjcules du màle, que nous sélectionnons en bouts de deux à trois centimètres dans le sens transversal, en mème temps que nous les fendons sur la longueur. Un testicule est mélangé avec les ceufs, tandis que l'autre est placé dans un bol d'eau. Aussitơt les ceufs sont versés dans ce bol, petit à petit, en ayant soin d'effectuer lo mélange au fur et à mesure du déversement. Il y a manifestement une grande quantité de laitance, car l'enu devient d'un blanc laiteux. Nous attendons onq minules, puis nous lavons les cufs à plusieurs reprises et les répartissons dans des bocaux. Au bout d'une heure très peu sont devenus blancs. Nous observons qủe ces oufs n'adhèrent pas au verre des bocaux, tandis que ceux obtenus de femelles vivantes à la Pisciculture, se collent dès qu'ils sont immobiles. C'est donc que le mucus qui les entourait a disparu ou a perdu ses propriétés adhé. sives.

Je mème jour, ì la fin de la soirée, de l'eau prise dans la Vilaine, est vidée directement, sans transition préalable dans la pièce, dans deux bocaux. Aussitôt beaucoup d'oufs deviennent blancs. Il est manileste que l'eau de la Vilaine étant plus froide que celle qui se trouve dans l'appartement, la variation brusque de température a provocuć une mortalité considérable sur les oufs. Par la suite, l'eau de la Vilaine ayant síjourné préalablement une heure ou deux dans la pièce, avant d'être utilisée, aucune mortalité n'a plus été constatée. Le 20 Mars ces ouls sont envoyés à la Pisciculture. Malheureusement un accident de transport a entraîné leur perte. On peut donc conclure, de celte manipulation, que l'opération de fécondation artificielle peut se faire sur des sujets morts. Il sera intéressant de déterminer jusqu'à quel moment, après la mort, les oufs et la laitance conservent encore leur vitalité. Nous pensons, l'an prochain, effectuer de nouvelles expériences en même temps qu'essayer de con- 
server et de transporter à sec, les oufs et la laitance, dans des conditions déterminées.

Le 16 Mars, nous avons également rapporté de la Pisciculture 65 ceuts de Brochets prélevés sur ceux qui ont été fécondés le i5. Ils sont conservés dans un bol, l'eau étant renouvelée une fois par jour. L'incubation s'effectue normalement et cinq aufs sculcment périssent. Le 23 Mars se produit la première éclosion, c'est-a-dire au bout d'une huitaine de juurs. L'eau, dans le bol, est à une température de $12^{\circ}$. L'éclosion se poursuit ensuite jusqu'au 27 Mars où clle est complètement terminée, c'est-à-dice le douziène jour.

Certains alevins paraissent avoir quelques difficultés à sortir de l'ocut. Nous les répartissons dans deux bols. Dans le premier nous disposons quelques brindilles de plantes d'appartement et rien dans le second. Dans le premier bol les alevins s'accrochent aux plantes par la ventouse qu'ils portent à la place de la bouche. Dans le second, ils rẹstent couchés sur le fond, tout en cherchant, par moments, à g'accrocher à quelque chose. 1ls y réussissent parfois en se tenant contre la paroi du bol on à des poussières très tenues qui flottent à la surface de l'eau. Tousefois, nous remarquons que les alcvins qui restent couchés sur le fondi du bol se développent aussi normalement que les autres. La résorption de la vésicule s'effectue de la mème manière, et les pertes, au total, sont insignifiantes, 2 à 3 sujets. Le samedi 5 Avril, la résorption de la vésicule étint très asancée chez tous les alevins, nous les déversons dans le canal.

Les 17 el 20 Mars, Je garde-pisciculteur opère la fécondation artificiclle sur trois femelles. Ne disposant que de màles ayant déjà été utilisés, il a sacrilié deux mâles avec la laitance desquels il a lécondé les coufs. La manipulation a été faite de la mème façon que dans les prcmières expériences. Le garie-pisciculteur a pris deux baquets sur le fond desquels il a disposé des mottes de gazon. Après avoir rempli ces baquets d'eau, il y a déversé les cuufs fécondés. Ceux-ci se sont immédiatement collés sur les herbes. P'endant les premiers jours, ces baquets ont été placés sous une goulotte d'où s'écoulait un filet d'eau. Ayant constalé que celui-ci amenait des dépôts nous l'avons supprimbé. Aucun soin n'a été pris pour les ceufs et aucun triage n'a été fait. Le 9 Avril, nous avons constaté que dans les deux baquets, il y avait des alevins de Brochets. Par conséquent l'incubation et réclosion s'étaient faites normalement. Ces alevins restent accrochés aux parois des baquets ou aux herbes. Dans cette manipulation, il est difficile de détermiṇer le pourcentage de réussite des ouls, leur surveillance n'étant pas commode.

Toutefois, cela montre que l'incubation, pour être réussie, ne nécessite ni une alínentation en eau abondante, ni des précautions compliquées. Il 
y a lieu de rapprocher cette opération de celle faite par un minotier du Mainc-et-Loire et que nous rappurtons ci-après.

Il y a quelques annćes, à l'époque de la fraie du Brochet, il eut la bonne fortune de trouver dans un tamburr, une femelle et un mâle qui étaient tellement à maturité, que les oufs ôl la laitance coulaient sans qua aucune pression sur le ventre soit nécessaire. Voyant cela, il prif un seau qu'il remplit à moitié d'eau. Tenant au-dessus de ce scau ses deux Brochets, it fit tomber dans l'eau, en mèmt temps oeufs et laitance. Cette dernière ètait abondante. L'eau ayant été agitée, le tout fut déversé dans un baquet, au fond duquel il avait placé des herbes prises dans la rivière. L'cau du baquel était renouvelée au moyen d'un pelit tuyau de caoutchouc lormant siphon. Au buut de dix à quinze jours, il vit un fourmillement d'alevins dans le baquet. Son opération de pisciculture artificielle avait donc fort bien réussi.

La températurc de l'eau, pendant la durée des essais, a élé de : le 16 Mars $6^{\circ}$; le le $17,9^{\circ} ;$ le $18,9^{\circ}$; du r9 au $24,9^{\circ}$; du 25 au $28,8^{\circ}$. Cette température était mesurée vers le milieu du jour. Le matin et le soir, elle était de quelques degrés inférieure ou supérieure.

De ces différentes expériences, on peut conclure que la fécondation artificiclle des coufs de Brochets peut the réussie sur des sujets vivants, ou morts depuis quelques heures. L'essenticl est davoir des femelles et des mâle à maturité. Si le nombre de mâles est insulfisant, le seul moyen d'obtenir assez de lailance est d'en sacrifier m ou plusieurs. La fecondation opérée à sec ne présente pas de diflicultés spéciales de manipulation. L'essentiel paraît être d'obtenir un múlange convenable des oufs et de la laitance et d'eviter de faire subir aux oculs, pendant les manipulations, des variations brusques de température.

L'opération de fécondation étant faite et les oufs lavés à plusieurs reprises, il convient de les déposer dans une carafe de Zug avec un faible courant d'eau. Brasser délicatement les coufs pour éviter qu'ils sc prennen! en paquets ou n'adhèrent aux parois. Au bout de quelques heures, une journée au plus, ils sont gonflés et ont perdu leur propriétẻ adhésive. On peut alors les déposer sur des claies d'incubation appropriées, dans des bacs d'alevinage absolument semblables à ceux qui sont ulilisés pour les wufs de Truites. Un très léger courant de ronouvellement d'eau est próférable. La surveillance, ainsi que l'enlèvement des oufs mauvais s'effectuent alors très facilement, comme dans la pisciculture de Truites. Après l'éclosion, la surveillance demeure facile. Au bout de $\delta$ à to jours, les jeunes alevins peuvent être déversés dans les cours d'eau ou en étangs d'élevage. Nous pensons pouvoir étudier, l'an prochain, la possibilité d'élever des alevins dans des bacs après résorption de la vésicule.

De cette manière, la pisciculture artificielle du Brochet s'effectue par des manipulations simples et pratiques comme pour la Truite. Il semble 
que l'on puisse placer dans un même bac d'alevinage $7^{5}$ à 100.000 auts sans crainte.

A notre avis, la carafe de Zug présente des inconvénients pour l'incubation pratique de grosses quantités d'ours. Le réglage du débil est, en effet, assez délicat. La possibilité de variation de ce débit, en cours d'in. cubation, peut provoquer soit l'amas deg ceufs dans le fond ou sur les cỏtés de la carafe, ou, au contraire, leur expulsion à l'extérieur. Dans les deux cas, cette circonstance est défavorable. De plus, le triage dẹ oufs mauvais est difficile, ou, en tout cas, ne peut être fait pratiquement sur de grandes quantités. Enfin, les expériences ci-dessus montrent qu'il n'esl pas utile d'avoir un courant d'eau continu et abondant, pís plus qu'une agritation constanle des oufs pendant toute la durée de l'incubation.

Nous allons maintenant examiner, à la lumière de nos observations, faites depuis plusieurs années, ce qui peut se passer dans la naturc. Il ne s'agit là que d'une hypothèse que nous n'avons pas encore été en mesure de vérifier au moment du frai des Brochets. A notre avis, à l'époque lavorable, les femelles sc rendent sur les frayères suivies des mâles. Y at-t-il plusicurs males pour chaque femelle?

ll nous paraît impossible d'énoncer une règle générale. L'n màle peut suffire pour une femelle. S'il y a plus de mâles que de femelles, alors plusieurs mâles vont avec une mème femelle. Ln mème màle peut, vraisemblablement, participer à la fécondation des ceufs de plusieurs femelles successivement. Quoinguil en soit, la fẹmelle prête à pondre se rend sur le lieu choisi, les mâles restent à proximité. Elle expulse quelques cufts (sans doute plusieurs centaines). Aussitòt, le mâle accourt et lâche sa laitance qui se mélange à l'eau où viennent d'être plongés les cufs. Ceux-ci tombent lentement et se collent aux lierbes, plantes, gràre à leur mucus. S'ils tombent sur la vase du fond ils périront étouflés. L'incubation se passe et arrive l'heure de la naissance. La queue du petit poisson sort la première. Elle s'agitc avec vivacité, tirant petit à petit de la coquille, Ic corps, la vésicule, la lète. Nous avons pu faire cette observalion, au moment de l'éclosion des cuís que nous avions dans des bols, à Rennes. Livré à lui-mème, le nouveau-né nage par saccades et cherchẹ un appui qu'il trouve dans les brindilles, plantes siluées à proximité. Il reste là, accroché par sa ventouse et attend la résorption de sa vésicule. S'il ne s'accroch! pas, le courant, mềme très faible, l'ẹntraîne, il roule sur le fond et meurt, ou a de grandes chances de périr. En pisciculture artificielle, le mucus est un inconvénient Il $n$ 'est pas utile puisque l'ceuf reste sur un fond par. failement propre. L'accrochage de l'alevin, après l'éclosion, ne parait pas non plus nécessaire. Il peut très bien rester allongé sur la claie d'incubation parfaitement propre et qui ne saurait le blesser.

Si les renseignements, qui nous ont élé donnés par des pécheurs, sont exacts, le Brochet affectionne, pour frayer, les rigoles remplies par les 
eaux d'inondation. Ce fait parait confirmé par le suivant. En aval de Rennes, la Vilaine traverse une grande région de marais, immédiatement en amont de Redon. Les hivers pluvieux, ces morais sont recouverts d'eau. Lorsque l'eau reste sur les marais jusqu'au printemps, ceriains auraient observé que l'alevin de Brochet est abondant. Au contraire, il y a peu d"alevins si la rivière reste dans son lit. En Juin I 44 , une remontée considérable de petits Brochets fût observée au premier barrage sur la Vilaine, en amont de Redon, à Màlon. Depuis, aucune remontée n'a été constatée de 1942 à 1946 inclus. Or, en $194 \mathbf{1}$, sans avoir de grandes crues, la Vilaine conserva un débit important, par suite des pluies, jusqu'à la mi-Avril el les marais de Redon restèrent inondés longtemps. Cette circonstance ne s'est pas renouvelée de 1942 à 1946 . Elle se reproduit à nouveau à l'heure actuelle si l'hypothèse précitée est exacte, il devrait y avoir remontée d'alevins de Brochets, à Mâlon, à partir de Juin prochain. Nous ne manquerons de faire les observations utiles.

Rennes, le 12 Avril 194\%. 